\title{
The efficacy and safety comparison of PD-1/PD-L1 antibody, chemotherapy and supportive treatment for pretreated advanced esophagogastric cancer: a network meta-analysis
}

\author{
Chuan Liu ${ }^{1,2 \#}$, Wancong Wang ${ }^{1 \#}$, Jiahui Yang ${ }^{3 \#}$, Pan Song ${ }^{4}$, Fuhao Li ${ }^{1,2}$, Bin Liu ${ }^{1,2}$, Jinpeng Li ${ }^{1,2}$, \\ Huimei $\mathrm{Xu}^{5}$, Yang $\mathrm{Mi}^{1,2}$, Youcai Tang ${ }^{2,6}$, Pengyuan Zheng ${ }^{1,2}$ \\ ${ }^{1}$ Department of Gastroenterology, The Fifth Affiliated Hospital of Zhengzhou University, Zhengzhou, China; ${ }^{2}$ Marshall B.J. Medical Research \\ Center of Zhengzhou University, Zhengzhou, China; ${ }^{3}$ Department of Gastroenterology, The First Affiliated Hospital of Xi'an Jiaotong University, \\ Xi'an, China; ${ }^{4}$ Department of Urology, West China Hospital of Sichuan University, Chengdu, China; ${ }^{5}$ Department of Gastroenterology, The Second \\ Affiliated Hospital of Lanzhou University, Lanzhou, China; ${ }^{\circ}$ Department of Children's Rehabilitation Medicine, The Fifth Affiliated Hospital of \\ Zhengzhou University, Zhengzhou, China \\ Contributions: (I) Conception and design: C Liu, W Wang, J Yang; (II) Administrative support: Y Mi, Y Tang, P Zheng; (III) Provision of study \\ materials or patients: F Li, B Liu, J Li, H Xu; (IV) Collection and assembly of data: C Liu, W Wang, J Yang; (V) Data analysis and interpretation: C \\ Liu, W Wang, J Yang, P Song; (VI) Manuscript writing: All authors; (VII) Final approval of manuscript: All authors. \\ \#These authors contributed equally to this article. \\ Correspondence to: Prof. Pengyuan Zheng, MD, PhD. Department of Gastroenterology, The Fifth Affiliated Hospital of Zhengzhou University; \\ Marshall B.J. Medical Research Center of Zhengzhou University, Kangfu Street No. 3, Zhengzhou, China. Email: medp7123@126.com.
}

Background: Immunotherapy is important for the treatment of esophagogastric cancer. The purpose of this study is to compare the efficacy and safety of PD-(L)1 antibody, chemotherapy, and supportive treatment in the management of pretreated advanced esophagogastric cancer.

Methods: The randomized controlled trials were identified by searching electronic databases including PubMed, Cochrane Library and Embase database. The network meta-analysis (NMA) was carried out using software R 3.3.2. Main outcomes including overall survival (OS), progression-free survival (PFS), all grades and serious treatment-related adverse events (TRAEs) were extracted and analyzed. The ranking results for all outcomes were performed to identify the best treatments.

Results: Seven high-quality RCTs involving 1,891 patients were taken into analysis. Compared with supportive treatment, PD-(L)1 antibody and chemotherapy both had a significantly longer OS time. Chemotherapy could obvious improve PFS than supportive treatment, but it had more all grades and serious TRAEs than PD-(L)1 antibody and supportive treatment. No significant difference was found in other comparisons. The probabilities of rank plot showed that PD-(L)1 antibody was the best in the outcome of OS. Chemotherapy ranked first in PFS and ranked last in all grades and serious TRAEs.

Conclusions: According to our results, PD-(L)1 antibody had excellent survival benefits and tolerable TRAEs for pretreated advanced esophagogastric cancer. It might be a suitable potential choice, especially for patients with high PDL1 CPS or with gastroesophageal junction cancer.

Keywords: Esophagogastric cancer (EGC); PD-(L)1 antibody; chemotherapy; network meta-analysis (NMA)

Submitted Dec 29, 2019. Accepted for publication Apr 07, 2020.

doi: $10.21037 /$ apm-19-670

View this article at: http://dx.doi.org/10.21037/apm-19-670 


\section{Introduction}

Esophagogastric cancer (EGC) remains an important health issue, of which gastric cancer (GC) ranks fifth most prevalent cancer globally, with an increase of around 1 million cases in 2018, and esophageal cancer (EC) is the seventh, with 0.57 million diagnoses cases in 2018 (1). Gastroesophageal junction cancer (GEJC) is a type of lethal malignancy at the junction between the esophagus and stomach. Even though EGC have improved due to the early screening and extensive local excision (2), many EGC patients still diagnosed at advanced malignancy. First-line chemotherapy comprising platinum and fluoropyrimidine (3) significantly extends overall survival (OS) by close to seven months for advanced EGC patients (4), but most patients continued to have disease progression after treatment. As for secondor third-line therapy, chemotherapy also has been widely used in the clinic for many years (5), including paclitaxel, docetaxel, irinotecan single or combined treatment (6). In addition, HER-2 antibody trastuzumab and VEGFR-2 antibody ramucirumab also applied as second or third line treatments for EGC patients (7-9).

In recent years, with the close relationship between the development of tumors and immune escape (10), the immune checkpoint pathway is critical in cancer treatment (11), such as programmed death-1 (PD-1) (12). The PD-1 receptor, expressed mainly on activated T-cells (13), is specifically bound to the PD-1 ligand1 (PD-L1) on the tumor cell to cause $\mathrm{T}$-cell apoptosis and promote neoplastic growth (14,15). Therefore, PD-1 antibody (anti-PD-1) can restore antitumor immune response by blocking the PDL1 signaling pathway (16). Moreover, overexpression of PD-L1 in GC (17) makes inhibition of PD-1 pathway a reasonable target for advanced EGC patients. It has become an effective treatment option for a variety of cancers, and anti-PD-(L)1 have demonstrated broad antitumor activity in early trials $(14,18)$. Recent studies have also shown that anti-PD-(L)1 play an active role in the treatment of pretreated advanced EGC (19-21).

Notably, the anti-PD-(L)1, including pembrolizumab, nivolumab and avelumab, were approved in the United States and Europe for the treatment of patients who have already been treated for advanced EGC recently (22). However, it is unclear which of the anti-PD-(L) 1 and chemotherapy has more benefit for the treatment of EGC. Therefore, we performed this network meta-analysis (NMA) to evaluate the difference of anti-PD-(L)1, chemotherapy and supportive treatment, and to find out the optimal choice for pretreated, advanced EGC.

\section{Methods}

\section{Search strategy}

Two independent the investigators searched online databases, such as PubMed, Embase and the Cochrane Library, from their inception to July 1, 2019. The keywords and related synonym including "advanced esophagogastric cancer", "gastric cancer", "esophageal cancer", "PD-1", "programmed death 1", "pembrolizumab", "nivolumab", "avelumab", "chemotherapy", "docetaxel", "paclitaxel", "irinotecan", "RCT", etc. were applied in the search strategy. References to related articles, such as metaanalysis, comments and other styles, was also manually searched. The qualifications of these retrieved documents were carefully checked using the EndNote software. Irrelevant studies were excluded by carefully looking at their title, abstract and even the full text.

\section{Selection criteria}

In general, the studies meeting the following criteria were considered eligible for inclusion: (I) patients were diagnosed as pretreated advanced gastric cancer or esophageal cancer; (II) interventions of the trails at least included anti-PD-(L)1 or chemotherapy; (III) randomized controlled trials.

The trials meeting the following exclusion criteria were considered ineligible: (I) Besides anti-PD-(L)1 or chemotherapy treatment, patients adopted with other therapies such as vaccine therapy and radiotherapy; (II) sufficient data of necessary information was not provided.

\section{Data extraction and quality assessment}

The extracted data were as follows: NCT number, the first author, publication year, journal, study phase, interventions of experimental and control groups, the sample size of each group and relevant clinical outcomes. The main outcomes included OS, progression-free survival (PFS), all grades treatment-related adverse events (TRAEs) and serious TRAEs. Serious TRAEs were defined as grade 3-5 TRAEs.

Two authors independently assessed the methodological qualities. For each included study, the following criteria were assessed and assigned a grade of low, medium or high risk bias: random sequence generation, allocation concealment, blinding of participants and personnel, 

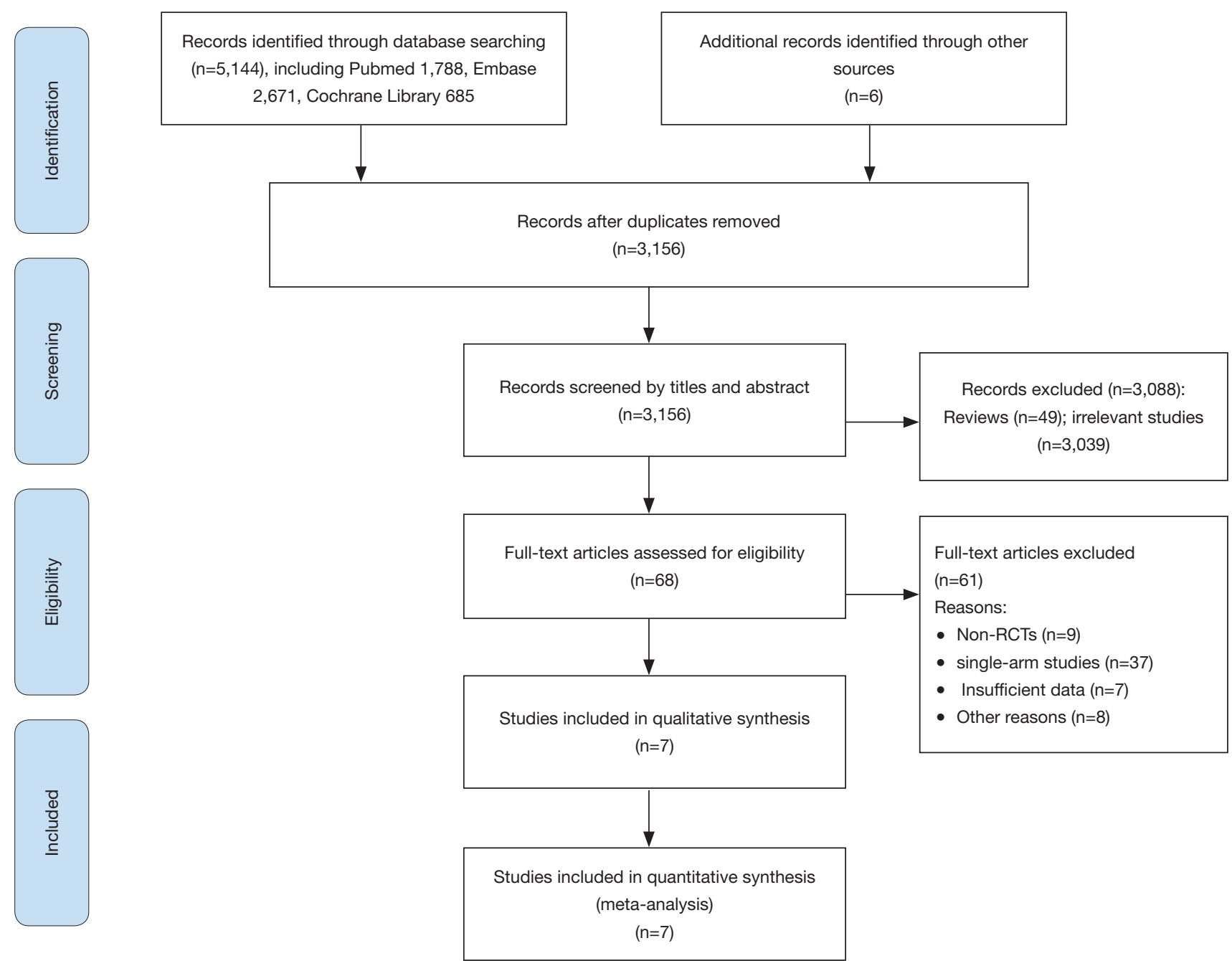

Figure 1 PRISMA flow diagram of the study selection process for network meta-analysis.

blinding of outcome assessment, incomplete outcome data, selective reporting and other bias. Any discrepancies between the two reviewers were regularly resolved by consulting with a third reviewer.

\section{Data synthesis and analysis}

Our NMA was carried out using R 3.3.2 software based on the Bayesian framework model, compared by both direct and indirect evidences. The pooled hazard ratio (HR) and $95 \%$ credible intervals (CIs) were used to estimate the two outcomes of OS and PFS. Dichotomous data, such as AEs, were calculated with odd risks (OR) and 95\% CI. A random-effects model was used to calculate the evidence inconsistency. The relative ranking results of the different approaches were presented as the probabilities. Node splitting was used to evaluate consistency. Subgroup analysis was performed according to PD-L1 combined positive score (CPS) and cancer type.

\section{Results}

\section{Baseline characteristics of the studies included}

The flow diagram of the article search and screening was shown in Figure 1. In total, 7 (23-29) RCTs including 1,891 patients were enrolled into our analysis. Baseline characteristics of selected articles were summarized in Table 1. The methodological quality of all included studies was 
Table 1 Main characteristics of the included studies in network meta-analysis

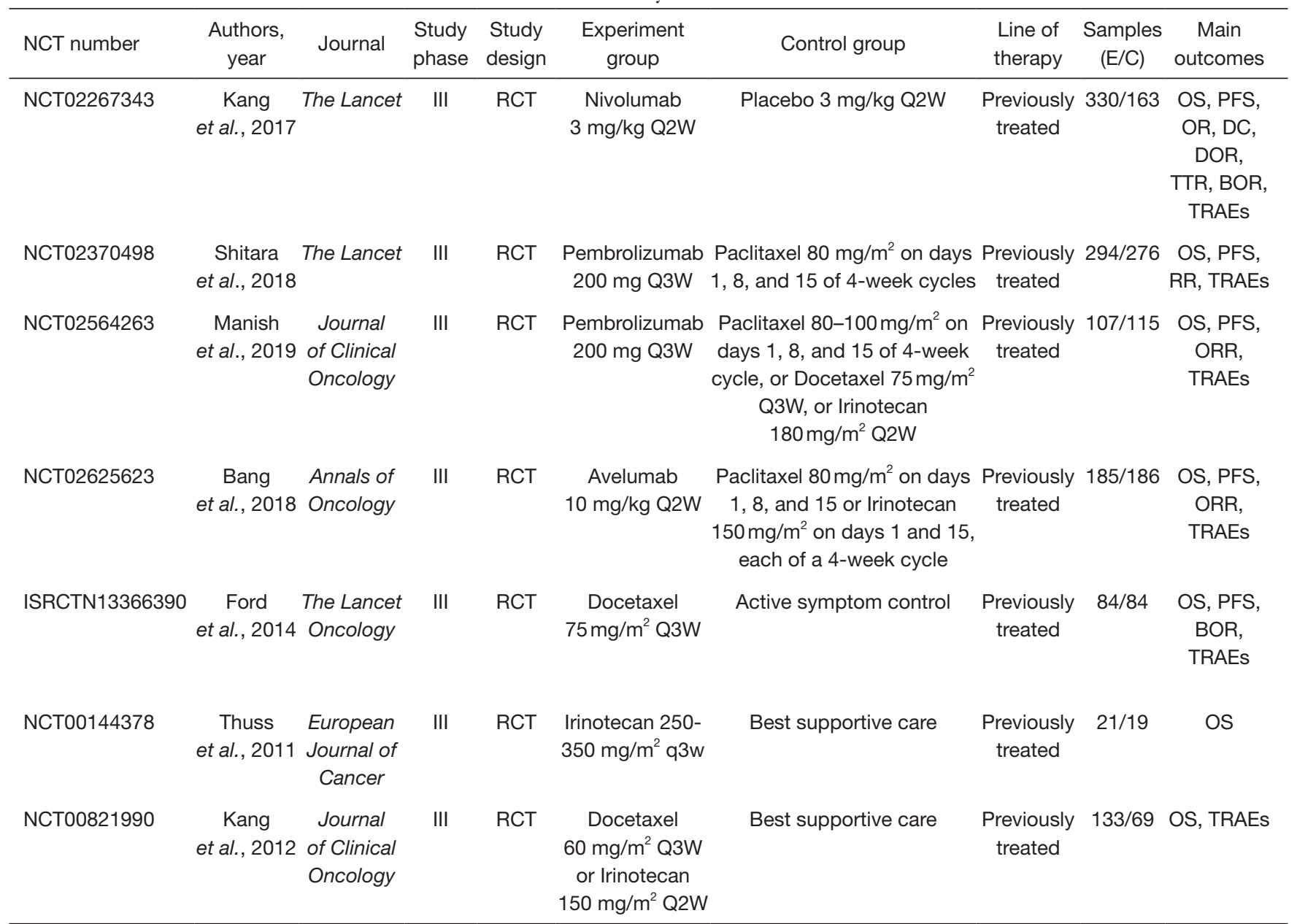

OS, overall survival; PFS, progression-free survival; OR, objective response; DC, disease control; ORR, objective response rate; DOR, duration of response; TTR, time to response; BOR, best overall response; TRAEs, treatment-related adverse events.

estimated. All 7 trials articles were randomized. The riskof-bias assessment of six studies were illustrated in Figure 2. The evidence network of three interventions were displayed in Figure 3.

\section{NMA of OS}

All 7 studies provided the HR value and 95\% CI, recruiting of 1891 patients. The NMA results of OS were shown in Table 2. Compared with supportive treatment, antiPD-(L) 1 and chemotherapy had a significant longer OS time [anti-PD-(L) 1 : HR $=0.59,95 \%$ CI $(0.42,0.80)$; chemotherapy: $\mathrm{HR}=0.66,95 \% \mathrm{CI}(0.49,0.86)]$. However, no significant difference were found between anti-PD(L) 1 and chemotherapy $[\mathrm{HR}=0.89,95 \%$ CI $(0.69,1.2)]$.
Besides, the ranking results showed that anti-PD-(L)1 ranked 1 st $(85.56 \%)$, chemotherapy ranked 2 nd $(85.23 \%)$ and supportive treatment ranked 3rd (99.20\%). Among these interventions, ranking 1 st and $3 \mathrm{rd}$ had the longest and the shortest OS time, respectively. The results of ranking analysis were presented in Figure $4 A$ and Table 3. In ranking plots of Figure 4, the lightest gray meant the first ranking, and the black meant the third ranking.

\section{NMA of PFS}

Three articles with 1,259 patients reported the HR value and $95 \%$ CI for PFS. The NMA results of PFS were presented in Table 2. Chemotherapy had a significant longer PFS $[\mathrm{HR}=0.41,95 \% \mathrm{CI}(0.18,0.94)]$ than supportive 


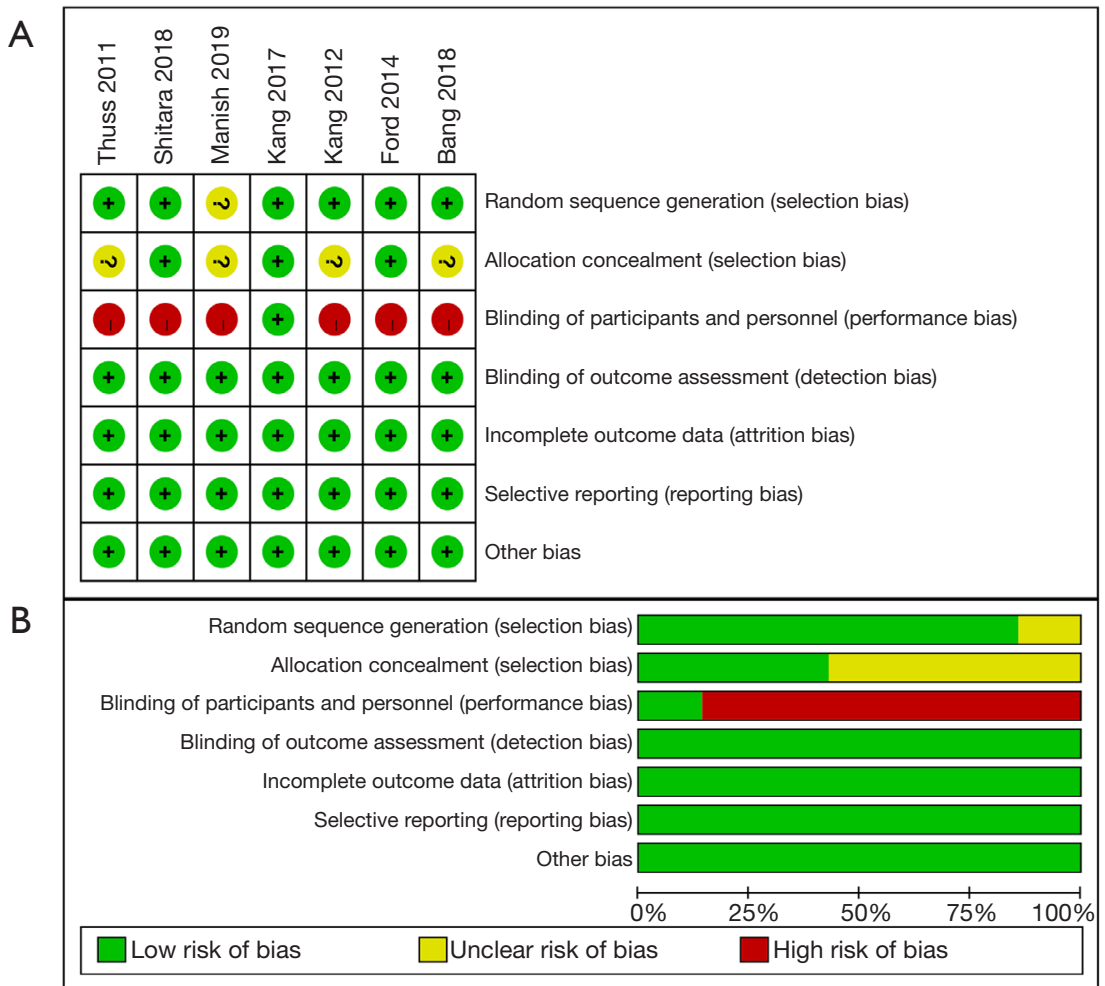

Figure 2 Risk of bias graph and summary of the included RCTs: (A) reviewers' judgements about each risk of bias item for eligible studies and (B) the judgements about each risk of bias item presented as percentages across all eligible studies.

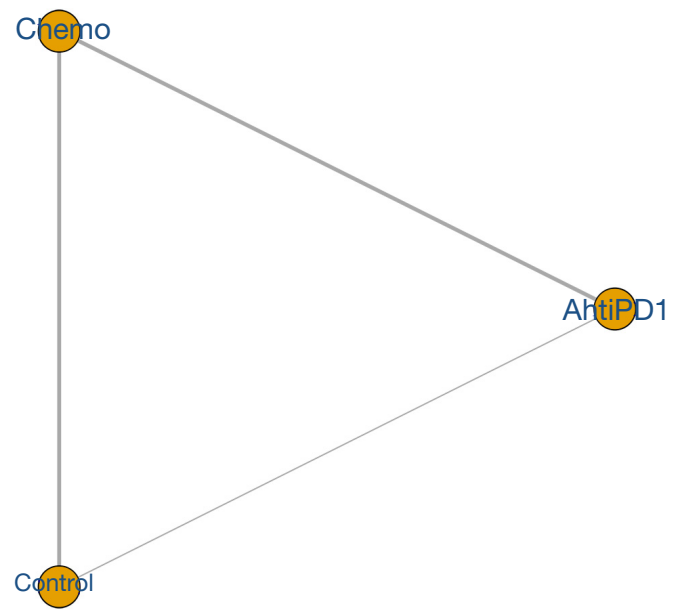

Figure 3 Network geometry of three interventions.

treatment. There were no obvious differences in the comparisons of anti-PD-(L)1 and chemotherapy. The probabilities of rank plot were as follows: chemotherapy ranked 1st (90.83\%), anti-PD-1 rank 2nd (94.23\%) and supportive treatment ranked 3rd (94.07\%). This ranking result were illustrated in Figure $4 B$ and Table 3.

\section{NMA of all grades TRAEs}

Three studies included with 1,422 patients described all grades TRAEs. The AE rates for anti-PD-(L)1, chemotherapy and supportive treatment were $47.77 \%$, $80.13 \%$ and $26.70 \%$, respectively. The NMA results showed that all grades TRAEs induced by chemotherapy were obvious higher than that of anti-PD-(L) 1 and supportive treatment [anti-PD-(L)1: OR $=3.79,95 \% \mathrm{CI}$ (2.41, 5.98); supportive treatment: $\mathrm{OR}=7.69,95 \% \mathrm{CI}(3.57$, 16.67)]. And more all grades TRAEs were observed in the anti-PD-(L)1 compared to supportive treatment [OR $=2.04,95 \%$ CI $(1.09,3.85)$ ] (Table 2). The results of rank plot analysis were showed in Figure $4 C$ and Table 3, which showed that supportive treatment ranked 1st (86.79\%), anti-PD-1 ranked 2nd (86.14\%) and chemotherapy ranked 
Table 2 Network meta-analysis results of treatment comparisons

\begin{tabular}{|c|c|c|c|}
\hline Treatment & Anti-PD-(L)1 & Chemo & Control \\
\hline vs. anti-PD-(L)1 & 1 & $1.1(0.87,1.5)$ & $1.7(1.2,2.4)$ \\
\hline vs. chemo & $0.89(0.69,1.2)$ & 1 & $1.5(1.2,2.1)$ \\
\hline vs. control & $0.59(0.42,0.80)$ & $0.66(0.49,0.86)$ & 1 \\
\hline vs. anti-PD-(L)1 & 1 & $0.68(0.42,1.1)$ & $1.7(0.84,3.3)$ \\
\hline vs. chemo & $1.5(0.90,2.4)$ & 1 & $2.5(1.1,5.7)$ \\
\hline vs. control & $0.6(0.31,1.2)$ & $0.41(0.18,0.94)$ & 1 \\
\hline \multicolumn{4}{|c|}{ All grades TRAEs, OR (95\% Cl) } \\
\hline vs. control & $2.04(1.09,3.85)$ & $7.69(3.57,16.67)$ & 1 \\
\hline \multicolumn{4}{|c|}{ Serious TRAEs, OR (95\% Cl) } \\
\hline vs. anti-PD-(L)1 & 1 & $3.73(2.41,5.79)$ & $0.4(0.17,0.93)$ \\
\hline vs. chemo & $0.27(0.17,0.41)$ & 1 & $0.11(0.04,0.28)$ \\
\hline vs. control & $2.5(1.08,5.88)$ & $9.09(3.57,25)$ & 1 \\
\hline
\end{tabular}

3 rd $(96.24 \%)$.

\section{NMA of serious TRAEs}

As for serious TRAEs, 4 articles with 1,644 patients were taken into analysis. This NMA results showed that chemotherapy had an obvious more serious adverse events than the anti-PD-(L)1 and supportive treatment [anti-PD(L)1: OR=3.73, 95\% CI (2.41, 5.79); supportive treatment: $\mathrm{OR}=9.09,95 \% \mathrm{CI}(3.57,25)]$. Anti-PD-(L)1 was associated with more serious TRAEs than supportive treatment $[\mathrm{OR}=2.5,95 \%$ CI $(1.08,5.88)]$ (Table 2). Furthermore, probabilities of rank plots were followed as: supportive treatment ranked 1st (88.37\%), anti-PD-(L)1 ranked 2nd $(87.45 \%)$ and chemotherapy ranked 3 rd $(96.71 \%)$ (Figure $4 D$ and Table 3).

\section{Subgroup analysis}

Subgroup analysis based on PDL1 CPS and cancer type showed that anti-PD-(L)1 could significantly extend OS than supportive treatment for patients with GEJC [HR $=0.42,95 \%$ CI $(0.20,0.90)]$. No obvious differences were detected in other comparisons in terms of improving OS in Table 4. But it shows in the ranking result that anti-PD-(L)1 ranked 1 st in OS in patients with PDL1 CPS $\geq 1$ or with EC and GEJC (Figure 5 and Table 5).

\section{Consistency and convergence analysis}

The node-splitting analysis was carried out to assess whether direct and indirect evidence in agreement. No significant inconsistency was found among the various treatments. That indicated that the consistency assumption was accepted. There was no significant publication bias among the included studies by the funnel plots. In addition, the potential scale reduction factor was limited to 1 , and the research had good convergence efficiency.

\section{Discussion}

In the past decade, esophagogastric cancer was the second most common cause of cancer-related death globally, with an estimated 1.6 million newly diagnosed and 1.25 million deaths per year (30). After first-line chemotherapy, some patients still have a risk of disease progression and 

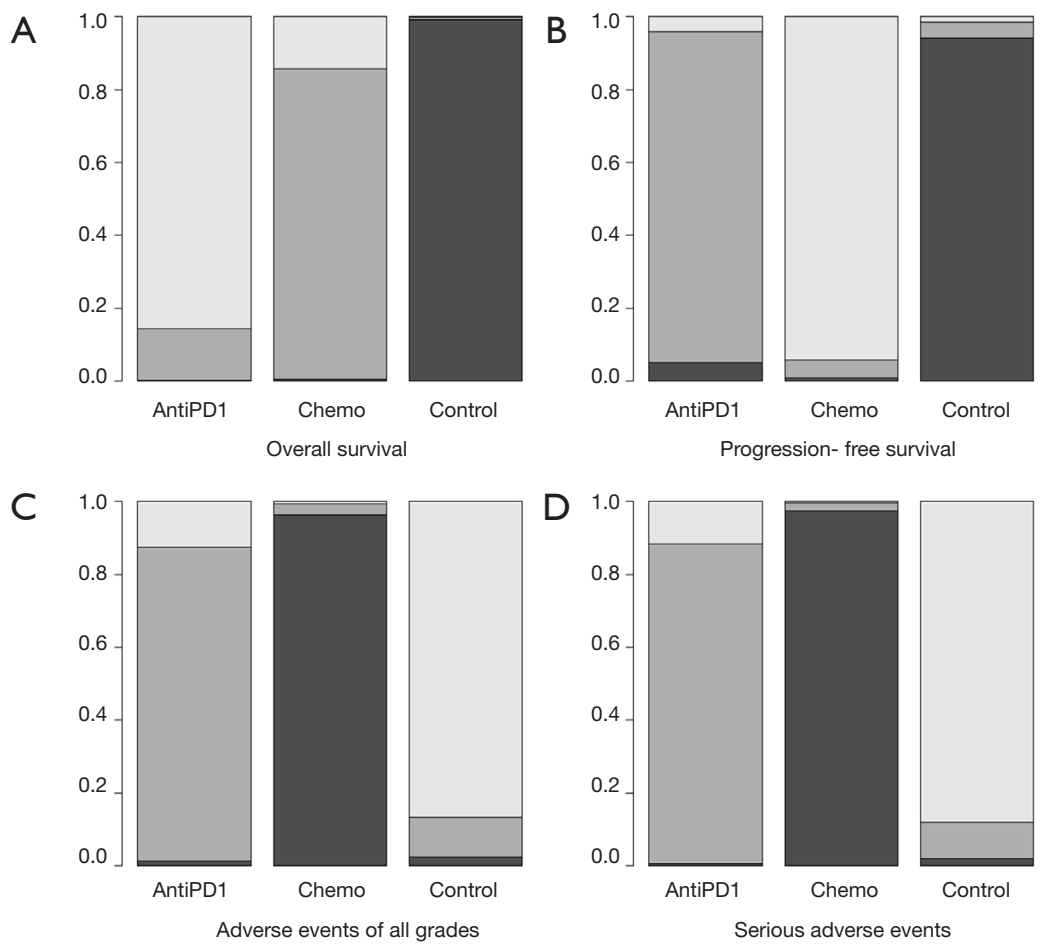

Figure 4 The relevant rank plots based on probabilities of interventions: (A) OS; (B) PFS; (C) all grades TRAEs; (D) serious TRAEs. OS, overall survival; PFS, progression-free survival; TRAEs, treatment-related adverse events.

Table 3 Rankings based on simulations

\begin{tabular}{lcccc}
\hline Endpoints (\%) & Ranks & Anti-PD-(L)1 & Chemo & Control \\
\hline OS & Rank 1 & 85.56 & 14.30 & 0.14 \\
& Rank 2 & 14.11 & 85.23 & 0.66 \\
& Rank 3 & 0.33 & 0.47 & 99.20 \\
PFS & Rank 1 & 4.16 & 94.23 & 1.61 \\
& Rank 2 & 90.83 & 4.85 & 4.32 \\
& Rank 3 & 5.01 & 0.92 & 94.07 \\
All grades TRAEs & Rank 1 & 12.49 & 0.72 & 86.79 \\
& Rank 2 & 86.14 & 3.04 & 10.82 \\
& Rank 3 & 1.37 & 96.24 & 2.39 \\
& Rank 1 & 11.23 & 0.40 & 88.37 \\
& Rank 2 & 87.45 & 2.89 & 9.66 \\
& Rank 3 & 1.32 & 96.71 & 1.97 \\
\hline
\end{tabular}

Rank 1 is the best; rank 3 is the worst. have a poor prognosis. For many years, chemotherapy, as traditional treatment, has been the most widely used for advanced EGC. Recently, immunotherapy, especially antiPD-(L)1, have been introduced as a relatively new treatment paradigm for advanced EGC patients. The therapeutic efficacy has been examined at different phases currently. In this study, we analyzed systematically the efficacy and safety of anti-PD-(L)1 and chemotherapy in pretreated advanced EGC patients.

In our NMA, 7 high-quality RCTs recruiting 1,891 patients were included in the analysis. The efficacy was assessed by the outcomes of OS and PFS, and the safety was evaluated in the outcome of all grades and serious TRAEs. We observed that, (I) Anti-PD-(L)1 could significant extend OS than supportive treatment. Anti-PD-(L)1 ranked first in OS and second place in PFS. In safety outcomes, antiPD-(L)1 was ranked 2nd following supportive treatment. (II) Anti-PD-(L)1 showed no significant difference with 
Table 4 Network meta-analysis results of OS in subgroup analysis

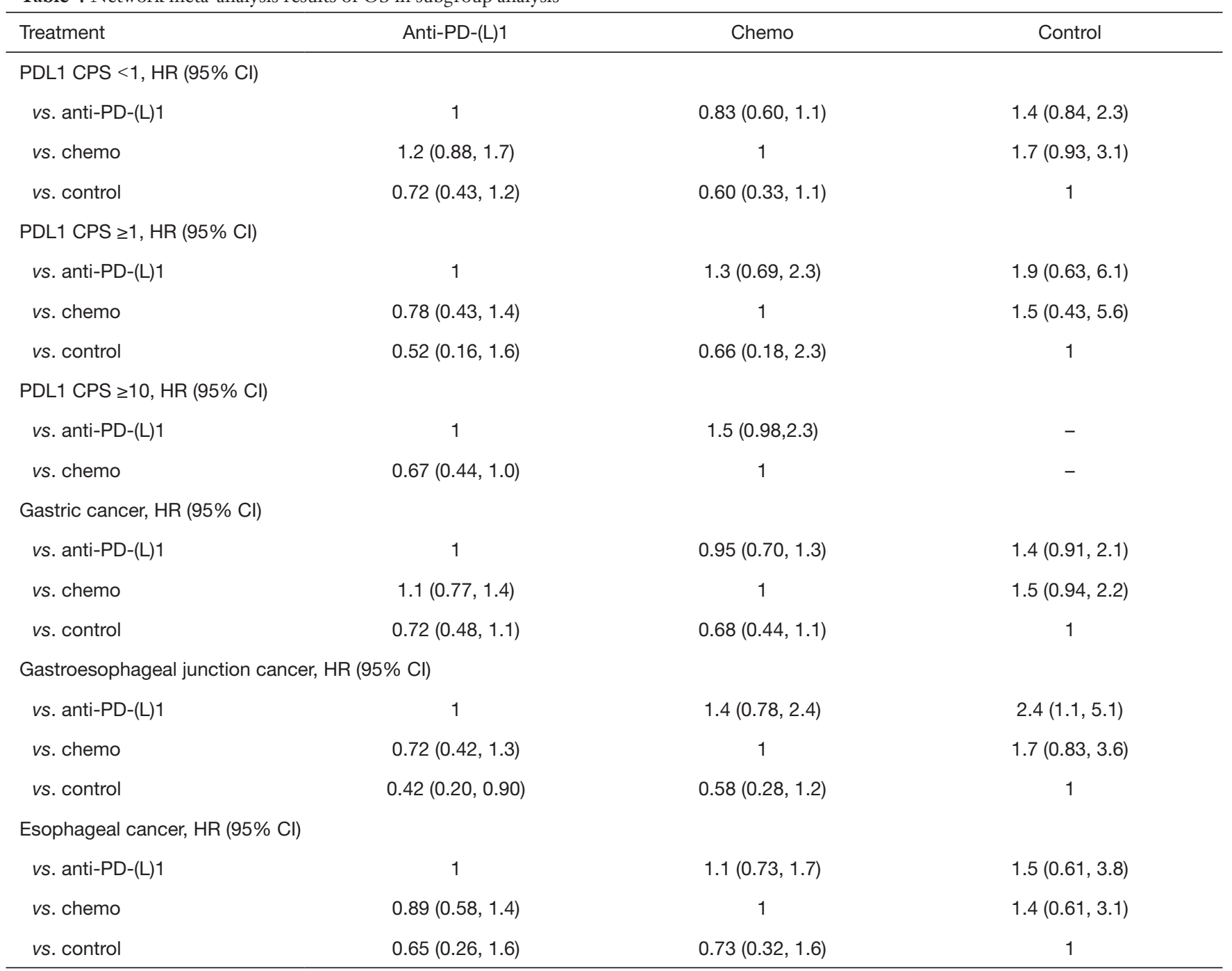

chemotherapy in OS and PFS, but anti-PD-(L)1 were associated with an obvious decrease in all grades and serious TRAEs compared with chemotherapy. According to the ranking plot results anti-PD-1 seemed to be more effective and safer than chemotherapy excepting PFS. (III) Chemotherapy could significant improve OS and PFS than supportive treatment. Nevertheless, the incidences of all grades and serious TRAEs of chemotherapy were higher than that of supportive treatment.

On the OS, some previously published reports have come to with our results to similar conclusions. Kang et al. (24) analyzed 493 patients, and revealed that anti-PD-1 could significant improve OS than supportive treatment for advanced GC patients, with a median OS of 5.26 months with anti-PD-1 versus a median OS of 4.14 months with control group. Ford et al. (27) concluded that chemotherapy had a longer OS time than supportive treatment. Similar results were seen in other studies $(28,29)$. Shitara et al. (25) found that the median OS of anti-PD-(L) 1 and chemotherapy were 9.1 months and 8.3 months respectively in patients with a PD-L1 CPS $\geq 1$, which showed they were not significantly different. Bang's study (23) reached similar conclusions. However, Shah et al. (26) reported that antiPD-(L)1 could have a statistical improvement in median OS than that of chemotherapy in patients with CPS $\geq 10$. This might be due to the higher PD-L1 CPS patients, the better its immunogenicity and response to anti-PD-(L)1 treatment, due to the high neoantigen burden and PD- 
A

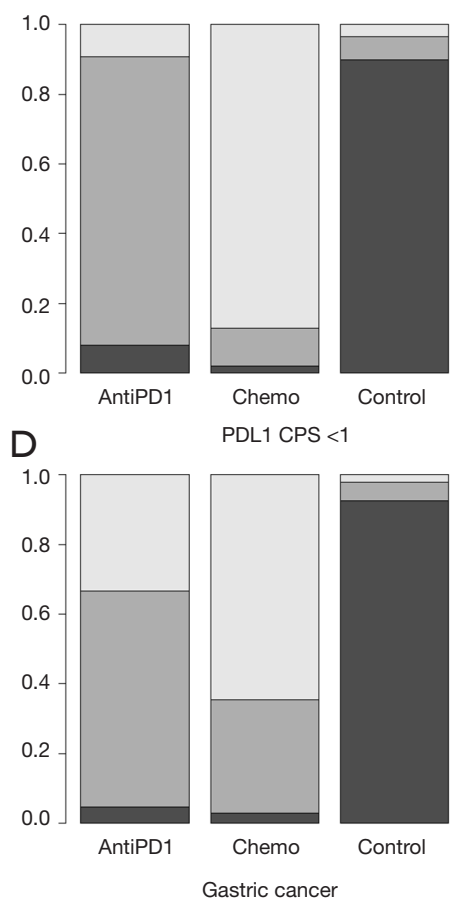

B

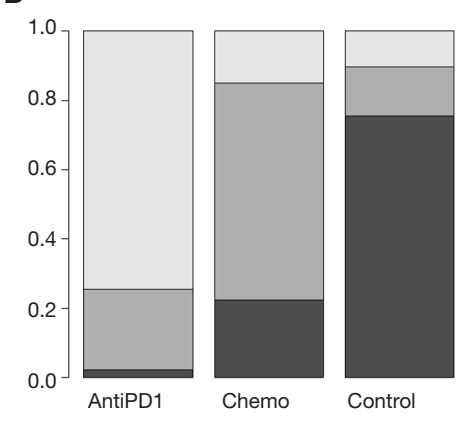

E

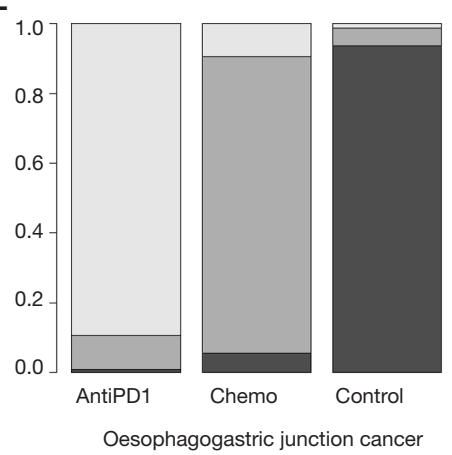

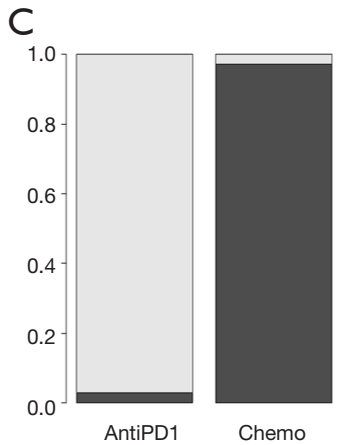

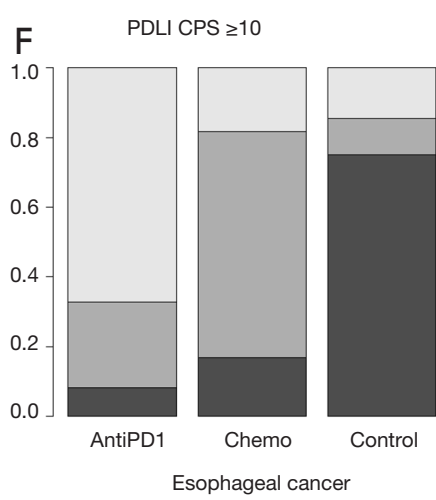

Figure 5 The relevant rank plots of subgroup analysis.

L1 expression $(31,32)$. The relationship between higher expression of PD-L1 and better therapeutic effect for antiPD-(L)1 was also found in the trial (20) and some studies in other tumor types $(33,34)$. For PFS, Shitara et al. (25) concluded that the lack of a PFS benefit with anti-PD-1 compared with chemotherapy. Similar results were found in Shah's study (26). Even Bang et al. (23) found chemotherapy had longer PFS than anti-PD-L1. So in terms of increasing PFS, anti-PD-(L)1 was not as significant as chemotherapy. According to the ranking results, anti-PD-(L) 1 had the longest OS and chemotherapy had the longest PFS.

As for the outcome of all grades and serious TRAEs concerned, we did find significant differences in these comparisons. Several previous trials $(27,29)$ also proved that the TRAEs was more frequent in chemotherapy group than the supportive treatment group. Even through anti-PD-(L)1 was associated with more TRAEs than the supportive treatment, its safety in advanced EGC patients were tolerable and manageable (24), and consistent with findings in patients with other advanced solid tumors $(35,36)$. Shitara et al. (25) found that anti-PD-(L)1 was less toxic (serious TRAEs, $14.2 \%$ for anti-PD-1 vs. $34.8 \%$ for chemotherapy) compared to chemotherapy $(23,26)$.
Compared with chemotherapy in the RCTs, anti-PD-(L)1 could significantly decrease the risk of common TRAEs, such as fatigue, anemia, asthenia, nausea, rash, pruritus, diarrhea, AST increased, neutropenia and leukopenia. This may be speculated that chemotherapy could damage epithelium-derived cells, while anti-PD-(L)1 may not. So for patients with poor basic function, in order to avoid side effects, we can properly consider the use of anti-PD(L)1. However, anti-PD-(L)1 are associated with increased incidence of immune-related AEs, such as pneumonitis and thyroid disease, which requires clinicians to pay more attention to potential TRAEs when using anti-PD-(L)1. As for supportive treatment, there are minimal AEs, such as infusion-related reactions and fatigue, because not treated with specific drugs. In a meta-analysis (37), it was demonstrated that anti-PD-(L)1 might be more effective and safer than the chemotherapy, which is consistent with our research.

PDL1 expression is considered a good biomarker. Subgroup analysis based on PDL1 CPS and cancer types were carried out to explore which patients are more likely to benefit. Anti-PD-(L)1 could significantly extend OS than the supportive treatment for patients with GEJC. 
Table 5 Rankings based on simulations of OS in subgroup analysis

\begin{tabular}{lcccc}
\hline Endpoints (\%) & Ranks & Anti-PD-(L)1 & Chemo & Control \\
\hline PDL1 CPS <1 & Rank 1 & 9.16 & 87.54 & 3.30 \\
& Rank 2 & 82.83 & 10.38 & 6.79 \\
& Rank 3 & 8.01 & 2.08 & 89.91 \\
PDL1 CPS 11 & Rank 1 & 74.47 & 14.62 & 10.91 \\
& Rank 2 & 23.24 & 62.22 & 14.54 \\
& Rank 3 & 2.29 & 23.16 & 74.55 \\
PDL1 CPS $\geq 10$ & Rank 1 & 97.14 & 2.86 & - \\
& Rank 2 & 2.86 & 97.14 & - \\
Gastric cancer & Rank 1 & 33.27 & 64.58 & 2.15 \\
& Rank 2 & 62.13 & 32.50 & 5.37 \\
& Rank 3 & 4.60 & 2.92 & 92.48 \\
Gastroesophageal & Rank 1 & 89.36 & 9.41 & 1.23 \\
junction cancer & Rank 2 & 9.73 & 85.05 & 5.22 \\
& Rank 3 & 0.91 & 5.54 & 93.55 \\
Esophageal cancer & Rank 1 & 67.15 & 18.25 & 14.60 \\
& Rank 2 & 24.75 & 64.94 & 10.31 \\
& Rank 3 & 8.10 & 16.81 & 75.09 \\
\hline
\end{tabular}

Rank 1 is the best; rank 3 is the worst.

But this study did not analyze the significant differences in other comparisons, which might be limited by the number of documents included. However, according to the ranked chart, anti-PD-(L)1 seemed to be more suitable for patients with high PDL1 CPS or with EC and GEJC. Patients with low PDL1 CPS or with GC, anti-PD-(L)1 did not show a clear advantage over chemotherapy. Previous studies have found that some tumor cells can escape immune recognition and destruction by expressing PDL1. Anti-PD-(L)1 can restore the tumor-suppression effect of $\mathrm{T}$ cells by blocking the PDL1 signaling pathway. So for immunotherapy, patients with tumors harboring PD-L1 expression exhibited higher responses than tumors without PD-L1 expression, so that they have better efficacy. As for cancer types concerned, different tissue types have different immune infiltration, so that they respond differently to immune checkpoint inhibitors, which is worthy of further discussion.

To the best our knowledge, this is the first NMA to compare anti-PD-(L)1, chemotherapy and supportive treatment for pretreated advanced EGC with direct and indirect evidences. Nevertheless, our study still has certain limitations. Firstly, availability of good data, only 7 highquality RCTs were taken into analysis, so it might not being convincing enough to perform a comprehensive NMA. Secondly, the supportive treatment were not identical, including placebo, active symptom control or best supportive care, which might interfere with the results. Thirdly, due to the lack valid data in included RCTs, some other outcomes such as objective response (OR) and disease control (DC) were not analyzed. This might also effect our evaluation.

In conclusion, anti-PD-(L) 1 and chemotherapy could significant improve OS than supportive treatment. Chemotherapy could increase PFS, but it was associated with all grades and serious TRAEs. Considering ranking results, anti-PD-(L)1 might be the optimal potential choice for pretreated advanced EGC, especially for patients with high PDL1 CPS or with GEJC. However, because of the limitations of this NMA, more high-quality researches are necessary to assess the options further.

\section{Acknowledgments}

Funding: The National Natural Science Foundation of China (No. 81370494 and No. 31471330) funded this manuscript. And the registered PROSPERO number was CRD42019142497.

\section{Footnote}

Conflicts of Interest: All authors have completed the ICMJE uniform disclosure form (available at http://dx.doi. org/10.21037/apm-19-670). The authors have no conflicts of interest to declare.

Ethical Statement: The authors are accountable for all aspects of the work in ensuring that questions related to the accuracy or integrity of any part of the work are appropriately investigated and resolved.

Open Access Statement: This is an Open Access article distributed in accordance with the Creative Commons Attribution-NonCommercial-NoDerivs 4.0 International License (CC BY-NC-ND 4.0), which permits the noncommercial replication and distribution of the article with the strict proviso that no changes or edits are made and the original work is properly cited (including links to both the 
formal publication through the relevant DOI and the license). See: https://creativecommons.org/licenses/by-nc-nd/4.0/.

\section{References}

1. Bray F, Ferlay J, Soerjomataram I, et al. Global cancer statistics 2018: GLOBOCAN estimates of incidence and mortality worldwide for 36 cancers in 185 countries. CA Cancer J Clin 2018;68:394-424.

2. Zhang X, Li M, Chen S, et al. Endoscopic Screening in Asian Countries Is Associated With Reduced Gastric Cancer Mortality: A Meta-analysis and Systematic Review. Gastroenterology 2018;155:347-354.e9.

3. Shen L, Shan YS, Hu HM, et al. Management of gastric cancer in Asia: resource-stratified guidelines. Lancet Oncol 2013;14:e535-547.

4. Wagner AD, Syn NL, Moehler M, et al. Chemotherapy for advanced gastric cancer. Cochrane Database Syst Rev 2017;8:CD004064.

5. Ajani JA, D'Amico TA, Almhanna K, et al. Gastric Cancer, Version 3.2016, NCCN Clinical Practice Guidelines in Oncology. J Natl Compr Canc Netw 2016;14:1286-312.

6. Hironaka S, Ueda S, Yasui H, et al. Randomized, openlabel, phase III study comparing irinotecan with paclitaxel in patients with advanced gastric cancer without severe peritoneal metastasis after failure of prior combination chemotherapy using fluoropyrimidine plus platinum: WJOG 4007 trial. J Clin Oncol 2013;31:4438-44.

7. Fuchs CS, Tomasek J, Yong CJ, et al. Ramucirumab monotherapy for previously treated advanced gastric or gastro-oesophageal junction adenocarcinoma (REGARD): an international, randomised, multicentre, placebocontrolled, phase 3 trial. Lancet 2014;383:31-9.

8. Wilke H, Muro K, Van Cutsem E, et al. Ramucirumab plus paclitaxel versus placebo plus paclitaxel in patients with previously treated advanced gastric or gastro-oesophageal junction adenocarcinoma (RAINBOW): a double-blind, randomised phase 3 trial. Lancet Oncol 2014;15:1224-35.

9. Bang YJ, Van Cutsem E, Feyereislova A, et al. Trastuzumab in combination with chemotherapy versus chemotherapy alone for treatment of HER2-positive advanced gastric or gastro-oesophageal junction cancer (ToGA): a phase 3, open-label, randomised controlled trial. Lancet 2010;376:687-97.

10. Dunn GP, Bruce AT, Ikeda H, et al. Cancer immunoediting: from immunosurveillance to tumor escape. Nat Immunol 2002;3:991-8.

11. Pardoll DM. The blockade of immune checkpoints in cancer immunotherapy. Nat Rev Cancer 2012;12:252-64.

12. Chinai JM, Janakiram M, Chen F, et al. New immunotherapies targeting the PD-1 pathway. Trends Pharmacol Sci 2015;36:587-95.

13. Ribas A. Tumor immunotherapy directed at PD-1. N Engl J Med 2012;366:2517-9.

14. Topalian SL, Hodi FS, Brahmer JR, et al. Safety, activity, and immune correlates of anti-PD-1 antibody in cancer. N Engl J Med 2012;366:2443-54.

15. Keir ME, Butte MJ, Freeman GJ, et al. PD-1 and its ligands in tolerance and immunity. Annu Rev Immunol 2008;26:677-704.

16. Iwai Y, Hamanishi J, Chamoto K, et al. Cancer immunotherapies targeting the PD-1 signaling pathway. J Biomed Sci 2017;24:26.

17. Cancer Genome Atlas Research Network. Comprehensive molecular characterization of gastric adenocarcinoma. Nature 2014;513:202-9.

18. Brahmer JR, Tykodi SS, Chow LQ, et al. Safety and activity of anti-PD-L1 antibody in patients with advanced cancer. N Engl J Med 2012;366:2455-65.

19. Janjigian YY, Bendell J, Calvo E, et al. CheckMate-032 Study: Efficacy and Safety of Nivolumab and Nivolumab Plus Ipilimumab in Patients With Metastatic Esophagogastric Cancer. J Clin Oncol 2018;36:2836-44.

20. Fuchs CS, Doi T, Jang RW, et al. Safety and Efficacy of Pembrolizumab Monotherapy in Patients With Previously Treated Advanced Gastric and Gastroesophageal Junction Cancer: Phase 2 Clinical KEYNOTE-059 Trial. JAMA Oncol 2018;4:e180013.

21. Muro K, Chung HC, Shankaran V, et al. Pembrolizumab for patients with PD-L1-positive advanced gastric cancer (KEYNOTE-012): a multicentre, open-label, phase 1b trial. Lancet Oncol 2016;17:717-26.

22. Fashoyin-Aje L, Donoghue M, Chen H, et al. FDA Approval Summary: Pembrolizumab for Recurrent Locally Advanced or Metastatic Gastric or Gastroesophageal Junction Adenocarcinoma Expressing PD-L1. Oncologist 2019;24:103-9.

23. Bang YJ, Ruiz EY, Van Cutsem E, et al. Phase 3, randomised trial of avelumab versus physician's choice of chemotherapy as third-line treatment for patients with advanced gastric or gastrooesophageal junction cancer: primary analysis of JAVELIN Gastric 300. Ann Oncol 2018;29:2052-60.

24. Kang YK, Boku N, Satoh T, et al. Nivolumab in patients with advanced gastric or gastro-oesophageal junction cancer refractory to, or intolerant of, at least 
two previous chemotherapy regimens (ONO-4538-12, ATTRACTION-2): a randomised, double-blind, placebocontrolled, phase 3 trial. Lancet 2017;390:2461-71.

25. Shitara K, Ozguroglu M, Bang YJ, et al. Pembrolizumab versus paclitaxel for previously treated, advanced gastric or gastro-oesophageal junction cancer (KEYNOTE-061): a randomised, open-label, controlled, phase 3 trial. Lancet 2018;392:123-33.

26. Shah MA, Adenis A, Enzinger PC, et al. Pembrolizumab versus chemotherapy as second-line therapy for advanced esophageal cancer: Phase 3 KEYNOTE-181 study. J Clin Oncol 2019;37:abstr 4010.

27. Ford HE, Marshall A, Bridgewater JA, et al. Docetaxel versus active symptom control for refractory oesophagogastric adenocarcinoma (COUGAR-02): an open-label, phase 3 randomised controlled trial. Lancet Oncol 2014;15:78-86.

28. Thuss-Patience PC, Kretzschmar A, Bichev D, et al. Survival advantage for irinotecan versus best supportive care as second-line chemotherapy in gastric cancer--a randomised phase III study of the Arbeitsgemeinschaft Internistische Onkologie (AIO). Eur J Cancer 2011;47:2306-14.

29. Kang JH, Lee SI, Lim DH, et al. Salvage chemotherapy for pretreated gastric cancer: a randomized phase III trial comparing chemotherapy plus best supportive care with best supportive care alone. J Clin Oncol 2012;30:1513-8.

30. Global Burden of Disease Cancer Collaboration, Fitzmaurice C, Akinyemiju TF, et al. Global, Regional, and
National Cancer Incidence, Mortality, Years of Life Lost, Years Lived With Disability, and Disability-Adjusted LifeYears for 29 Cancer Groups, 1990 to 2016: A Systematic Analysis for the Global Burden of Disease Study. JAMA Oncol 2018;4:1553-68.

31. Le DT, Durham JN, Smith KN, et al. Mismatch repair deficiency predicts response of solid tumors to PD-1 blockade. Science 2017;357:409-13.

32. Asaoka Y, Ijichi H, Koike K. PD-1 Blockade in Tumors with Mismatch-Repair Deficiency. N Engl J Med 2015;373:1979.

33. Garon EB, Rizvi NA, Hui R, et al. Pembrolizumab for the treatment of non-small-cell lung cancer. N Engl J Med 2015;372:2018-28.

34. Balar AV, Castellano D, O'Donnell PH, et al. First-line pembrolizumab in cisplatin-ineligible patients with locally advanced and unresectable or metastatic urothelial cancer (KEYNOTE-052): a multicentre, single-arm, phase 2 study. Lancet Oncol 2017;18:1483-92.

35. Ferris RL, Blumenschein G Jr, Fayette J, et al. Nivolumab for Recurrent Squamous-Cell Carcinoma of the Head and Neck. N Engl J Med 2016;375:1856-67.

36. Valsecchi ME. Combined Nivolumab and Ipilimumab or Monotherapy in Untreated Melanoma. N Engl J Med 2015;373:1270.

37. Chen C, Zhang F, Zhou N, et al. Efficacy and safety of immune checkpoint inhibitors in advanced gastric or gastroesophageal junction cancer: a systematic review and meta-analysis. Oncoimmunology 2019;8:e1581547.
Cite this article as: Liu C, Wang W, Yang J, Song P, Li F, Liu B, Li J, Xu H, Mi Y, Tang Y, Zheng P. The efficacy and safety comparison of PD-1/PD-L1 antibody, chemotherapy and supportive treatment for pretreated advanced esophagogastric cancer: a network meta-analysis. Ann Palliat Med 2020;9(4):1770-1781. doi: 10.21037/apm-19-670 